\title{
¿Y el re-formador qué? Las competencias del formador La formación de profesores de matematicas. El caso de la UD
}

\author{
Jorge Orlando Lurduy Ortegón \\ Grupo Crisálida. jolurduy@udistrital.edu.co
}

\section{Resumen}

En este documento proponemos criterios y reflexiones para analizar las competencias de los formadores de profesores de matemáticas para desde allí presentar y analizar algunos aspectos de los procesos de formación de profesores de matemáticas desarrollados en el programa de Licenciatura En Educación Básica Con Énfasis En Matemáticas de la Universidad Distrital de Bogotá. Planteamos algunos de los requerimientos de formación, competencias para los formadores de profesores. Finalmente disponemos algunas implicaciones de lo anterior en el mejoramiento de la formación de profesores.

\section{Introducción}

Es evidente la importancia, multiplicidad e intensidad de los debates en tomo a procesos de reforma a los programas de formación de profesores. Parece también necesario y pertinente profundizar en las conversaciones sobre desde dónde habla el "reformador", auáles deben ser las competencias de los "formadores" que hagan plausible dicha reforma y cuáles las condiciones para entablar una "negociación" para una tal reforma.

Una forma de acción inicial en esta dirección es planteamos preguntas vitales, comunes y autorreferentes como ¿Porqué hacemos lo que hacemos?, ¿Qué importancia tiene lo que estamos haciendo, cuando lo hacemos, con quien lo hacemos, desde donde lo hacemos?, ¿Cómo lo hacemos?, estas son preguntas ontológicas, epistemológicas y metodológicas, referidas a este ritual de RE-FORMA ${ }^{1}$, pero básicamente son preguntas existenciales de lo humano.

En este documento, primero disponemos unas observaciones generales sobre las "competencias de los formadores", unas reflexiones en tomo al proceso, diseño configuración e implementación de la "nueva" propuesta de formación de formación de profesores en la perspectiva de los formadores, para desde allí reflexionar en tomo a la formación de profesores de matemáticas y de sus formadores, en el caso del programa de formación de Licenciatura En Educación Básica Con Énfasis En Matemáticas (LEBEM-UD) de la Universidad Distrital De Bogotá.

\footnotetext{
${ }^{1}$ No me parece irrelevante utilizar el término "re-forma", finalmente esta acción es siempre UNA conversación, es un ritual, es inaugural desde lo positivo de lo "nuevo", en el sentido de vuelto a comenzar; es permanente porque nunca se ha dejado de hacer en $\mathbf{n}$ años y futura pues los resultados de ella, "hoy", se sistematizaran y se seguirán trabajando para lo que sigue como evolución pedagógica (acreditación, investigación, extensión y cualificación académica). Es decir es una forma de reconocer el pasado, la continuidad y el azar de toda conversación. (a veces trascendental, compleja, necesaria y profunda; a veces superficial, simple, contingente, instrumental, y en ocasiones y en diferentes dimensiones: todas las Anteriores)
} 


\section{Las competencias ${ }^{2}$ de los formadores}

Dada la diversidad de concepciones y la complejidad del propósito de la posibilidad de conformación (re-forma) de un proyecto de formación de profesores de matemáticas, proponemos unos reconocimientos-acuerdos-condiciones básicos entre participantes (pedagogos, psicólogos, filósofos, sociólogos, antropólogos, licenciados en matemáticas, matemáticos), pues ellos deberán orientar las acciones e implementación de aualquier propuesta de re-forma y para ellos quizás estas sea el comienzo de una reflexión sobre una genealogía de las competencias profesionales de los formadores de profesores y de los educadores en general:

No podría existir conversación (pero tampoco educación, formación) conmigo-otros si no nos informamos sobre algunas de nuestras creencias; querer, reconocer y aceptar la intención de "interpretarnos" desde donde el otro habla.

Esto plantea la difíil paradoja del dialogo, ¿Cómo me separo de mi, hablo desde usted, sin dejar de ser yo mismo?, esta es una renuncia y una ganancia que nos toca vivir todos los días (sobre todo a formadores de profesores y a los profesores) y arya salida es que podamos sensibilizarnos, conocer $y$ degustar nuestra disposición al antagonismo, la complementariedad propios de los encuentro humanos.

El siguiente acuerdo es sobre la implicación de lo anterior, la consideración misma, la consideración por el otro-en el otro-con el otro, es decir aceptar el supuesto de una diversidad óntica, epistémica, y metodológica, es necesario reconocer, aceptar y asumir nuestro principio de conocimiento y aceptación de lo otro/del otro.

Partimos de la presunción de que queremos, podemos y nos implicamos ${ }^{3}$, complementaria y simultáneamente en la re-forma de algún programa de formación de profesores, en este sentido ella plantea unos "retos" que propongo sean tenidos en cuenta en la perspectiva de las competencias mencionadas anteriormente en tomo a la interpretación, el entendimiento y sus implicaciones, ellos serían:

Existe una cierta "comparabilidad" en las perspectivas ontológicas, epistemológicas (filosóficas e ideológicas) de quienes intervenimos en los procesos de re-forma, que en ocasiones, invisibiliza los mismos supuestos e imposibilita el diálogo constructivo.

Existe una cierta "no comparabilidad" en condiciones: investigativas, de producción académica en cantidad, tematicidad y temporalidad; políticas, ideológicas y sociales; en los planteamientos que se hacen, con las respectivas implicaciones de incomprensión, sesgo e interpretación.

La potencialidad, actualidad e importancia de los planteamientos de los participantes en el campo de la formación de profesores y sus implicaciones para la educación, ponen de presente un "cierto grado de mediación y comparabilidad" en la esfera filosófica (epistemológica, ontológica y metodológica), política e ideológica; en un lenguaje común; en las creencias y concepciones sobre

\footnotetext{
${ }^{2}$ Como participantes e interesados en el tema de la formación de los docentes, somos conscientes del profundo, intenso y largo debate nacional e internacional sobre el "problema" de las competencias, en la polisemia del término, sobre la procedencia e implicaciones de la política de formación por competencias, etc., en este escrito asumiremos una concepción pragmática y propositiva frente al concepto de competencia, en lo relacionado con la formación de profesores, lo pedagógico y lo didáctico de las matemáticas escolares, finalmente es un término que usamos en el programa de formación de profesores donde vivimos, en nuestra práctica de formación y en lenguaje cotidiano académico o cotidiano.

${ }^{3}$ En cualquier acepción, sentido, significado de la palabra competencia, si uno no quiere, no puede y no se implica no es "competente", no tiene la competencia, no es su interés, no usa el conocimiento, en ningún contexto, para nadie o ningún otro en lo comunicativo, discursivo, operativo, normativo, etc.

${ }^{4}$ Quiero, puedo y me implico en el uso del término "reto" atractor, irónico, y muy comprometido 
lo pedagógico y lo didáctico, sobre la formación y la formación de profesores, las matemáticas su enseñanza y aprendizaje.

La emergencia en nuestras intervenciones del falibilismo, pluralismo y diversidad en una reflexión requiere una competencia, ella es modal. Es posible la asociación, precisión, discriminación de los términos, proposiciones y argumentos de un diálogo constructivo en el marco de una "conversación"5: La "no comparabilidad", se refiere a los espacios discursivos relacionados, dialogantes y complementarios pero daramente diferentes; limitantes, condicionantes; "la comparabilidad" se refiere a espacios discursivos de sensibilidad, imaginación y creatividad.

Por otra parte están los hechos: el problema generalmente invisible (casi fantasmagónico) de los intereses, la historia, los afectos, los gustos, el futuro e intenciones laborales, económicos, de estabilidad, de reconocimiento, de política, de placer, de poder de quienes intervenimos en las conversaciones y que hacen parte de nuestra "competencia comunicativa negociadora" que nuestros profesores más tarde asumirán como parte de su profesionalidad como profesores.

Lo aul nos pone en las dimensiones comparables y no comparables en niveles (plásticos, maleables, difusos) de lo posible, lo fáctico y las consecuencias de los hábitos y regularidades, emergen, se expresan, aparecen, se presentan como:

1. Lo óntico en la posibilidad de la profesión ser profesor: identidad, autoafirmación, y autoreconocimiento (que es tan necesaria en los profesores como parte de su competencia profesional), se trata de la aceptación del significado de lo contingente, lo real y lo necesario de su proyecto de vida como profesores (de profesores); de reconocimiento, afirmación e identificación; de goce, disfrute y alegría; y que ellos se viven en los mundos de lo individual, colectivo y lo institucional.

2. Lo epistémico emergente de la interacción entre el saber y el creer: en el plano de lo físico, lo simbólico, y lo trascendente, una instancia de construcción, dialogo y complejización; de configuración, realización y cualificación; de memoria, encuentro y futurización; de reflexión, contraste y transformación; de inducción, deducción y abducción; y que ellos se viven en los mundos de lo individual, colectivo y lo institucional.

3. La metódica, la implicación caracterizada por las formas: de la conversación, de la interacción, del conflicto; del antagonismo, del dialogo y de la complementariedad; del mundo de las interpretaciones, de la intersubjetividad y de la retroacción; en el pluralismo y la diferencia, lo diverso. (complejidad y pragmatismo).

Los intereses, la historia, los afectos, los gustos, el futuro e intenciones del ser, del saber y del poder convierten nuestro ser, saber, actuación y su relación en algo que estoy llamando competencias $^{6}$, de manera pragmática y con el ánimo de convención.

He usado el término competencia para referime a retos (disposición, posibilidades), hechos (capacidades y destrezas, acciones e interacciones) y hábitos (habilidades, regularidades), en este sentido no creo conveniente, ni real poder "definirlas", pues ellas no son, las competencias, emergen. Ellas hacen parte de los puntos (región) de frontera epistémica, (no son ni endógenas ni

\footnotetext{
${ }^{5}$ Si se asume la postura "posmoderna" del fin de los relatos, de la inconmensurabilidad de los discursos, del relativismo, de la diversidad como reconocimiento de inferioridad, no podrá haber conversación, sólo monólogos disfrazados de dialogo.

${ }^{6}$ También es muy reconocida la libre y conveniente utilización del término COMPETENCIA en documentos de constituciones nacionales, políticas de estado en lo social, documentos oficiales de todos los gobiernos y en todas la reformas en lo educativo como: leyes, decretos, acreditación, estándares, indicadores, logros, evaluación, objetivos y todo cuánto se quiera controlar, cuantificar, medir. Propongo mirar las otras caras de la moneda COMPETENCIA, no uniformadora, no indicativa, no cuantificadora, no controladora, no predictiva, no universal, no determinada, no ordenada, no necesaria y trascender las posturas "reactivas y contestarías" frente al tema, él es global, y definitorio.
} 
exógenas, ni internas ni externas, ni individuales ni colectivas, ni espontaneas, ni determinadas) por tanto garantizan la continuidad entre las diferentes instancias de la realización de lo presente, son la mediación entre lo local y lo periférico.

Las competencias "van siendo" parte de lo fronterizo. Van emergiendo en una relación ónticoepistémica de tipo dialogal, creativa, indeterminada, sin límites concretos; implican un dialogo creativo, en ellas se alcanza creatividad, abducción, imaginación?.

A manera de articulación entre las perspectivas aquí asumidas para la "Competencia" y para efectos pragmáticos, aceptamos con Tejada (2007) la siguiente aproximación a la conceptualización de dicha noción.

.. se debe recalcar que una competencia es el producto de la interaccióndialéctica $y$ permanente entre la afectación, la acción y la reflexión, entendiéndose por reflexión la posibilidad de análisis, conceptualización, sistematización, procesamiento, teorización, inferencia, etc., y la acción como la posibilidad de desempeño, de hacer, de actuar, de ejecutar. De allí, que suponer que la sola refiexión es suficiente para lograr la formación de una competencia, o que la acción a ultranza la posibilite, es en opinión de este artíaulo una posición ingenua basada en visiones fragmentarias, reduccionistas $y$ mecanicistas. El interjuego dialéctico y permanente entre la afectación, la reflexión y la acción, podrá ser mejor entendido desde una perspectiva de la complejidad, la flexibilidad, la integración y la totalidad. $Y$ ese interjuego debe ser definido y caracterizado en la conjugación de todos loselementos, procesos, condiciones, etc., que constituyen una competencia. ( $p, 47)$.

A manera de Alto en el camino, algunas adaraciones frente a esta descripción pseudo ontológica de la competencia (el "Reto"):

1. Percibir la posibilidad (me dan ganas, me gusta, me afecta, me sensibiliza, planeo, diseño) en relación individual conmigo mismo y con mi historia, con mi proyecto

2. Describir y distinguir el "hecho" en el sentido de lo realizable, de las relaciones de actuación con los demás (el contexto, las personas, mi formación, mi historia, mi saber, mi experiencia, de gestionar mi vida, tomar opciones, decidir en el ya y el ahora) en relación con mi "quiero" y mi realización como humano

3. Pensar los "hábitos" como las regularidades y las consecuencias, las implicaciones, los resultados de mi acción, es la mediación entre los retos y los hechos pero que se convierte en un reto para volver a empezar desde lo emergente

Desde esta caracterización de las "competencias" proponemos hablar del tipo de formación para profesores de matemáticas y las agendas de investigación de esa formación identificadas por los investigadores y la investigación actual en el tema de la formación de los profesores de matemáticas

\footnotetext{
${ }^{7}$ Me parece serio y académico preguntarme sobre el contenido y el sustento filosófico, o pedagógico, o didáctico de afirmaciones tan usadas en libros, textos y documentos como "competencia colectiva", "competencia exógena", "competencia pedagógica", "competencia laboral", "competencia matemática", pues cada vez que se usan se deben invertir ríos de tinta explicando y aclarando desde dónde se habla, para quién, en qué contexto, en qué etimología del término. Potencia, acto, conocimiento, capacidad, destreza, habilidad, saber, comprensión, uso o todas las anteriores. Formamos, desarrollamos, construimos, descubrimos competencias y cada vez la confusión es mayor. La plasticidad del término es infinita y da para todo 


\section{La formación de profesores y de los formadores}

En LEBEM-CRISÁLIDA ${ }^{8}$ partimos de pretender formar al profesor como un profesional de la cultura, investigador, reflexivo, crítico, no segregador, que resuelve problemas sobre su propia práctica y desde una perspectiva constructiva crítica y compleja, esta formación la hemos realizado como un proceso sistemático y organizado mediante el aual los EPP se implican en su formación individual y/o colectiva, de forma cática y reflexiva asumen su opción de formación profesional como profesor de matemáticas para la educación básica.

Este tipo de formación la hemos propuesto para los formadores y profesores de los profesores en formación, nos parece consistente pretender re-formar la formación de profesores desde creencias que son acordes con las concepciones sobre la formación que se pretende, sobre la educación matemática, las matemáticas, los procesos de estudio, etc como los que enunciamos a continuación:

Para nosotros la reflexión y auto reflexión de la experiencia vivida como profesores y formadores de profesores nos posibilita la modelación de un programa y se convierte en una epistemología: el mundo en el cual vivimos no nos es ajeno, de manera necesariasomos observadores y observados simultáneamente en el hecho pedagógico y didáctico, es en el mundo de la investigación y la reflexión sobre la formación de profesores de matemáticas donde hemos construido nuestra experiencia pedagógica, formativa y formadora, didáctica e investigativa.

De acuerdo con Morín (2000) el conodimiento no es conocimiento si no es reflexión-acciónorganización, siguiendo a Bateson (1981) la epistemología es "La reflexión acerca de cómo determinados organismos conocen, piensan y deciden" y de acuerdo con Godino (2008) el significado de los objetos es el conjunto de prácticas discursivas, operativas y normativas de una persona o una institución frente a una situación problema puestas en relación y en contexto con las informaciones (epistemología).

Estas ideas, nos han ayudado a pensar y estudiar, los procesos formativos en el seno de los sistemas didácticos, en ellos los procesos de estudios sobre los objetos didácticos y en ellos las competencias de reflexión y análisis didáctico (tres objetos de estudio en la formación de profesores).

Para ello necesitamos, en primer lugar, de un contexto teónico constructivo, recursivo, activo, rico y diverso que nos ayude a favorecer didho estudio con el apoyo de los recursos epistemológicos: falibilidad, pluralismo, diversidad, pragmaticismo, reflexividad y complejidad. (Peirce, Lewis, Morín, Von Foster, Maturana, Navarro, Faema, Nubiola, Zalamea, Dussel...).

Pensamos y nos decidimos, en segundo lugar, por la complejidad del modelo del sistema didáctico, ello exige otro tipo de respuestas investigativas y de apuestas epistemológicas, ontológicas y metodológicas consistentes con los principios triádicos de la semiótica Peirceana y los del pensamiento complejo (Morín, 2000): dialógico (antagonismo y complementariedad), recursivo (retroacción causa-efecto, y hologramático (el todo está representado en la parte).

\footnotetext{
${ }^{8}$ En este documento comprometo mi postura profesional de formador de profesores de matemáticas. Aunque ella ha sido ampliamente debatida y compartida al interior del programa, es obvio pensar que no todos los seres humanos que interactuamos en él tenemos la misma y única forma de asumir idiosincráticamente el programa de LEBEM. Los planteamientos aquí enunciados hacen parte de los desarrollos personales y grupales en la investigación y conceptualización de la formación de profesores de los integrantes del grupo de investigación CRISÁLIDA.

${ }^{9}$ Sobre el tema de la formación y la formación de profesores la literatura es bastante profusa, y diversa, nosotros asumimos pragmáticamente este concepto de formación, conscientes de que él ameritaría un mayor detenimiento y profundización 
Tercero, en nuestra perspectiva explicativa e interpretativa de tipo relacional compartimos con Navarro (2000) arando enuncia la reflexividad y autoreflexividad de los sistemas observadorobservado. Para este autor un sistema es reflexivo en la medida en que en su seno se generan efectos reflexivos, entre la actividad del sistema objeto y la actividad objetivadora-significativa del sujeto, ello nos pone en la dirección de las relaciones y reflexiones pedagógicas y didácticas en educación matemática y en la formación de profesores de matemáticas.

Por último, pensamos y decidimos aquí por una epistemología relaciona ${ }^{10}$ es decir el estudio de los sistemas cuya materia prima es la relación entre objetos, eventos, hechos, procesos y fenómenos y una teoría del observador ${ }^{11}$ : ya que el acto educativo es una actividad social aparece la incertidumbre, la autoorganización y estas se relacionan con la dimensión social y comunicativa, Semiótica: algunos autores como Lahitte (1994), afirma:

"La cosmovisión ecológica, cibernética, sistémica (o como se llame) [...] requiere de diferencias de información que organice la energía material de tal modo que "la mente" (el pensamiento) pueda "corporizarse" y para eso necesita un observador. Necesita un contexto de significación donde sean significativas las diferencias "emergentes", en que las distinciones tengan sentido, es así como una epistemología relacional, brinda este contexto, para que las explicaciones y modelaciones tengan significación" (p. 20)

\section{Algunas consideraciones finales}

Las agendas y desarrollos actuales: La situación de los desarrollos teóricos y empíricos de los programas de formación actualmente planteada, nos ha confirmado en nuestra perspectiva de formación, por ejemplo Ponte y Chapman, (2007) en relación con la evaluación de programas de formación y su investigación sugieren trabajar en metodologías y agendas así: Trabajos individuales y estudios de trabajo en grupo; Estudios basados en el curríaulo; Visiones de la práctica docente; metodologías provenientes de disciplinas como la antropología y la estadística; Promover estudios sobre la "buenas" prácticas, condiciones sociales favorables, principal atención a otras instancias y espacios de realización del profesor como un profesional, de la complejidad de la relaciones en el "sistema didáctico", en los procesos de formación de profesores, las relaciones entre la práctica y la vida real y en la evaluación de las políticas de formación, en la integración de marcos teóricos y metodológicos.

Y Lester (2007), sobre investigación en formación y desarrollo profesional del profesor, a partir de la pregunta sobre la formación del profesor: ¿Cuáles son los propósitos de la formación de profesores? Algunas de las respuestas son: Desarrollo y formación de una visión compartida para la enseñanza y el aprendizaje de las matemáticas; Desarrollo y formación de un adecuado conocimiento de contenido matemático; Desarrollo y formación de una comprensión adecuada de cómo los estudiantes piensan y aprenden las matemáticas; Desarrollo y formación de un conocimiento de contenido pedagógico Desarrollo y formación de una comprensión adecuada del rol de la equidad y la igualdad en la formación matemática; Desarrollo y formación un sentido de si mismo y de identidad como profesor de matemáticas

Complementariedad, intersubjetividad y dialogo en la formación de profesores: Para los anteriores investigadores dichos propósitos son complementarios e interdependientes unos de

\footnotetext{
10 Esta epistemología relacional que tiene su origen en la teoría general de sistemas (Ashby, 1964), teoría de la comunicación (Wilden, 1979), la teoría general de sistemas (Bertalanfy, 1978), teoría de la complejidad (Morín, 1982), ), la cibernética de segundo orden (Von Foerster (1988), la Biología del conocimiento (Varela y Maturana, 1990).

${ }^{11}$ Remitimos al dásico articulo de Heinz Von Foerster "Notas para una epistemología de los objetos vivientes" (1974), donde enuncia 11 principios de la epistemología de los sistemas observadores que da origen epistemología relacional, de la reflexividad y la autoreferencia 
otros y le permiten al profesor saber qué hacer, cómo hacerlo y hacerlo con convencimiento y agrado por la profesión (emergencia de competencias). ¿Qué principios pueden ser usados para guiar el diseño de programas de formación de profesores de matemáticas? Implementación de aurríaulos induyentes, con métodos constructivos; Desarrollo de principios de trabajo colaborativo en redes; participación en investigación con otras universidades y centros académicos; Evaluación permanente, procesos de investigación curricular, clases, proyectos, actividades; Investigación, desarrollo, y énfasis en las prácticas matemáticas y en las prácticas docentes de profesores y estudiantes; desarrollo de las publicaciones de trabajos de estudiantes y profesores.

El profesor formador investiga: La idea de profesores investigadores no es reciente, ella se fundamenta en la necesidad de realizar cambios profundos en la práctica educativa y en el desarrollo del rol profesional del profesor. Esta perspectiva de presupone que si bien es cierto que se acepta la función docente como la más importante dentro del trabajo profesional, su mejoramiento no podrá darse sin una actitud critica, reflexiva y permanente de su práctica profesional. Como consecuencia de lo anterior hemos desarrollado tareas en los procesos de formación y de los profesores de los estudiantes para profesor que nos han permitido un mayor acercamiento a la investigación en el aula entendida como un camino que hace que el futuro profesor haya sido formado en prácticas docentes de un profesor (formador) que reflexiona sobre la acción y en la acción, en consecuencia, mejora su práctica, trabaja con criterios de rigurosidad, coherencia y consistencia y le es posible innovar a partir de la reflexión "en/para/sobre" su propia práctica como formador.

Nuestra apuesta es de un currículo como proyecto de investigación: Para nuestro caso, hemos elegido investigar nuestra propia práctica, en tanto nuestra propuesta curricular es ella misma un proyecto de investigación, hemos elegido cono principal instancia de realización profesional del profesor el aula y por tanto la denominada investigación en el aula de clase, con estudiantes para profesor que vivencien la investigación, la reflexión, el análisis didáctico, el desarrollo de competencias como las enunciadas más arriba, ya que consideramos que:

1. Nuestro currículo de formación pretende la formación inicial de profesores, en tal sentido, sólo pretendemos generar en el EPP una actitud, unos hábitos, disciplinas y motivaciones hacia la investigación.

2. El aula es el ámbito en el cual el profesor identifica sus problemas, reflexiona, teoriza y propone soluciones. El profesor como profesional autónomo debe ser quien genere su propio conocimiento mediante la reflexión de su práctica, apoyado en el trabajo colectivo con sus compañeros de institución educativa, en la medida en que participa del análisis, critica y confrontación de las experiencias profesionales y en la posibilidad de reconocer y valorar su trabajo como el de los demás.

3. Los problemas de investigación del profesor, surgen de las dificultades que se presentan durante la enseñanza en la escuela, así como de los proyectos interdisciplinarios que se pretendan desarrollar como proyecto educativo institucional. En el hacer cotidiano del profesor se vincula la teoría con la práctica, entendiéndola como un proceso de transformación de la realidad.

Uno enseña como le han enseñado: Por tanto aspiramos a transformar nuestras prácticas formativas con la pretensión de que nuestros estudiantes cambien las de ellos o en las que ellos fueron "alfabetizados en matemáticas", aceptamos con Juan francisco Aguilar que nos hemos propuesto como una innovación educativa en el campo de la formación de profesores de matemáticas para la educación básica y asumimos "El Reto" de haber sido acreditados de alta calidad por el CNA (2006) con estas características y por tanto de asumir la rendición de cuentas de tipo pedagógico y didáctico en el impacto y proyección de nuestros profesores y nuestros estudiantes en sus respectivas prácticas pedagógicas. 


\section{Bibliografía}

Bateson, G. (1989), Pasos hacia una ecología de la mente, planeta, Buenos Aires.

Godino, J. D. (2008), Marcos teóricos de referencia sobre la cognición matemática disponible en Internet (jgodino@ugr.es).

Lurduy, 0 (2009). El profesor investigador de su práctica. En: La formación del profesorado de matemáticas. Uno, Revista de didáctica de las matemáticas, № 51.

Morín, E (2000). Una mente bien ordenada. Seix Barral, Barcelona.

Ponte, P y Chapman, O. (2007). Past, present and future, Mathematics teacher's knowledge and practices En: Handbook Of Research On The Psycology Of Mathematics Education PME 1976-2006.

Sowder, J. (2007). The mathematical education and development of teachers. En: Second Handbook Of Research On Mathematics Teaching And Leaming, edited Frak K Lester, Jr.

Tejada, A. (2007). Desarrollo y formación de competencias: Un acercamiento desde la complejidad, Accion pedagógica No 16, Cali 